\title{
Les exclamatives de Mlle Pellaton
}

\author{
Frédéric Gachet et Gilles Corminboeuf \\ Universités de Fribourg et de Neuchâtel
}

\section{Contexte de la recherche}

Un courriel est à l'origine de cette étude ${ }^{1}$. Une étudiante de l'Université de Neuchâtel, occupée à rédiger, sous la direction des auteurs, un travail de séminaire sur le discours indirect libre dans La Table-auxcrevés (1929) de Marcel Aymé, nous l'avait adressé pour se renseigner au sujet des constructions nominales soulignées dans (1) et (2) :

(1) $\S$ Le beau soleil qu'il faisait dehors. Coindet alla prendre l'air.

- Bon Dieu qu'il fait bon, dit-il en pissant sur le fumier. [Aymé, La Table-aux-crevés]

(2) Coindet se plaignait de l'attitude de Legeai et des autres. Il redoutait des catastrophes. Truchot le rassurait :

- Pourquoi que tu te tourmentes. (...) Y a pas de raison que tu te fasses du souci. Laisse aller.

Coindet était réconforté. Le bon copain que c'était, Truchot. Il ne faisait pas de bruit, mais quelque chose arrivait à Coindet, il disait ce qu'il fallait dire, il faisait ce qu'il fallait faire. [ibid.]

L'étudiante faisait l'hypothèse que l'emploi de ces énoncés nominaux pouvait être attribué à la sphère énonciative du personnage, par opposition à des indices plutôt associés à celle du narrateur (les temps du passé, par exemple). Elle interprétait légitimement cette contradiction d'indices comme le révélateur d'un discours indirect libre. Son courriel nous demandait simplement si cette formulation était considérée comme typique de l'oral, et sous quel nom on la désignait.

Le statut de cette construction à l'égard de l'opposition entre oral et écrit nous semblait particulièrement délicat à déterminer. Les énoncés averbaux passent souvent pour des caractéristiques de l'oral spontané, ce que ne confirment généralement pas les corpus à disposition. Chez Marcel Aymé, il ne fait guère de doute que cette construction contrefait un «parler populaire » et fait écho à l'idiolecte d'Urbain Coindet. Elle appartient à l'image que se fait l'écrivain de l'oralité, sans être forcément un trait prototypique de l'oral spontané. Sa représentation réelle en français parlé reste relativement énigmatique pour nous, en l'absence d'une collection suffisante de données orales.

Quant au nom à donner à ces constructions, nous n'avions naturellement pas de réponse à fournir à notre étudiante. Nous avons d'ailleurs pris l'habitude de les désigner entre nous sous l'appellation de « relatives de Mlle Pellaton », ou " exclamatives de Mlle Pellaton », rendant ainsi un hommage sans cérémonie à l'étudiante qui nous y a rendus attentifs.

Quelques recherches ciblées - dans les travaux sur les énoncés averbaux, les relatives et l'exclamation nous ont confortés dans le sentiment que cette construction restait largement méconnue, justifiant qu'on entreprenne son étude.

\section{Etablissement du corpus et délimitation de la structure}

\subsection{Le corpus}

Le corpus servant à l'étude de cette construction a été collecté dans la banque de données Frantext, dans diverses œuvres littéraires (Belle du Seigneur de Cohen, Le Planétarium de Sarraute, entre autres), dans la presse écrite, etc. Les pointages effectués dans les corpus oraux en accès libre n'ont pas donné de résultats 
très probants. On peut penser que l'oral narratif, le genre le plus représenté dans ces corpus, n'est pas le terrain le plus propice à l'apparition de ces constructions. Par ailleurs, celles-ci se prêtent mal à une recherche automatique dans les corpus d'oral (sur quels segments baser la recherche ? ${ }^{2}$ ) Toutefois, cette structure nous semble parfaitement familière et bien attestée dans la communauté linguistique ; il nous est arrivé à plusieurs reprises pendant nos recherches d'en attraper l'une ou l'autre occurrence à la volée, v. (10) à (13), (20), (26) et (46).

\subsection{Les constructions étudiées}

La construction est constituée d'un SN défini, suivi dans la majorité des cas par une relative en que. Nous avons distingué deux groupes de constructions, selon que le SN est introduit par un article défini $(\S 2.2 .1)$ ou par un démonstratif $(\S 2.2 .2)$.

\subsubsection{SN défini}

Lorsque le SN est introduit par l'article défini, il peut comporter une épithète évaluative, à valeur aussi bien positive ${ }^{3},(3)$ à (6), que négative, (7) à (9) :

(3) Avec une corolle dans le dos il est tombé en Provence sur les arrières des lignes allemandes. Ça lui avait valu des breloques... Elles étaient sur le coussin rouge posé sur son cercueil... Oh ! la belle jambe que ça lui a fait ! Il les aurait sûrement troquées pour quelques mois de vie supplémentaires. [Boudard, Mourir d'enfance, 1995, $\mathrm{f}^{4}$ ]

(4) Elle a une façon de vous regarder droit, comme pour se demander si vous êtes celui qu'elle imagine, si vous l'êtes bien... tu deviens fou. Le beau parti que tu fais, avec tes cent vingt-cinq francs par an de la légion d'honneur et tes soixante-deux francs par mois de demi-solde ! [Pourrat, Les Vaillances, 1930, f]

(5) Le ravissant entretien que ce fut là ! [Milosz, L'Amoureuse Initiation, 1910, f]

$$
\text { - Oh ! Les charmantes poses }
$$

Que prit pour se lever l'enfant aux lèvres roses ! [Dierx, Poèmes et poésies, 1864, f]

(7) Les fâcheuses humeurs que vous avez là tous deux ! [Hugo, Notre-Dame de Paris, 1832, f]

(8) La piètre narratrice que je fais ! [Frapié, La Maternelle, 1904, f]

(9) - Oh ! le vilain mot que vous venez de prononcer là ! dit la jeune fille avec une petite moue ; est-ce qu'il y a des lettres importantes quand ce n'est pas à moi que vous écrivez ? [Gautier, Récits fantastiques, 1856, f]

Le SN peut aussi se présenter sans épithète :

(10) le BOL qu'elles avaient [oral, à la volée ; énoncé avec dépit par l'un des perdants d'une partie de cartes, à propos des gagnantes $]^{5}$

(11) euh mais la TEte que j'ai [oral, à la volée, 23.08.2011 ; devant le miroir]

(12) euh mais les sourires que tu me fais [oral, à la volée, 08.09.2011; à un bébé]

(13) putain le TEMPS qu'il lui faut quoi [oral, à la volée, 08.08.2011; un client à propos d'une vendeuse malhabile]

(14) Et puis hier... le mépris et l'horreur que m'inspiraient [sic] cette femme... mais que Dieu en ait pitié ! [Sue, Les Mystères de Paris, 1843, f] 
(15) Et ça a continué par terre avec l'explosion, coup sur coup en rafale, des munitions qui restaient dans la charge. Ça n'arrêtait plus : baoummm, blammmm, boouuheuum. Le potin que ça faisait... [Bayon, Le Lycéen, 1987, f]

La présence de la relative ne paraît pas indispensable à la structure. On pourrait imaginer par exemple que la formule exclamative de (9) se réduise à Oh! le vilain mot! tout comme celle de (15) à Le potin! Voici l'exemple d'une telle formulation minimale :

(16) - Chapeau, mon vieux, tu t'en es tiré comme un chef!

- Le coup de pot oui ! Le cul ! Le cul au carré ! [Degaudenzi, Zone, 1987, f]

Inversement, les SN de (16) auraient pu être complétés sans difficulté par une relative (le coup de pot que j'ai eu, oui!)

\subsubsection{SN démonstratif}

Lorsque le SN est introduit par un démonstratif, il se présente plus fréquemment sans épithète :

(17) Ce soupir que je me pousse intérieur ! [Boudard, La Cerise, 1963, f]

(18) Oh ! cette fadeur que mes vêtements éparpillent dans ma chambre ! Je me rappelle que j'aimais la verveine autrefois... Non, je ne me rappelle rien... [Frapié, La Maternelle, 1904, f]

(19) $-(\ldots)$ Il est charmant, Simon.

- Ah, je ne trouve plus. Cette manière qu'il a eu de se jeter à la tête de cette fille !... Je suis trop vieille, n'est-ce pas ? Demanda-t-elle. [Druon, Les Grandes familles, 1948, f]

La présence de l'épithète est cependant aussi attestée :

(20) et puis alors ça va + ce GROS dodo que tu as fait [oral, à la volée, 24.09.2011; à un bébé]

(21) Et puis elle a poussé sa fille vers Battaincourt. Mais lui, après cette tournée où il n'avait pas rencontré le regard d'un ami, il avait les yeux pleins de larmes, et il ne voyait rien : il a fallu lui mettre la fillette sur les genoux. Ce faux sourire qu'il a eu, en se penchant vers l'enfant de l'autre ! [Martin du Gard, Les Thibault, 1923, f]

Dans la variante avec démonstratif, comme dans celle mettant en jeu l'article défini, la structure peut se présenter sans la relative :

(22) Dans La Valse de Ravel, l'orchestre tangue comme sur une houle (ces motifs ondoyants aux violoncelles), les sonorités sont suggestives, mais là encore, on souhaiterait plus d'abandon. [presse, Le Temps, 10.03.11]

(23) Après porte Duronne, sur la nationale, il accélère. Cette vache de moteur ! Quelle mélodie sous le pied! Cette puissance ! C'est autre chose que sa Chambord 1958! [Thérame, Bastienne, 1985, f]

Les constructions à SN démonstratif sont courantes sans la relative, comme dans (22) et (23). Certaines paires minimales comme Ce temps qu'il fait! / Ce temps! versus Le temps qu'il fait! / ??Le temps ! suggèrent que les $\mathrm{SN}$ démonstratifs s'accommodent plus facilement de l'absence de la relative. Cela fournit déjà un indice d'un fonctionnement différent de ces exclamatives selon qu'elles sont introduites par l'article défini ou par le démonstratif (v. infra, § 4.3). 


\section{Description de la structure}

\subsection{SN défini, proposition relative et présupposition}

Dans nos constructions nominales, la relative est de type restrictif (ou déterminatif). On est en présence d'une description définie, le plus souvent sommaire, qui sert avant tout à ancrer le SN dans une situation particulière. La relative est souvent peu informative, du moins en ce qui concerne le verbe choisi : que c'est, que tu fais, que ça lui fait, que nous avons, etc. Ainsi, dans (2), elle paraît complètement superflue informativement (le bon copain que c'était). Le choix du verbe semble souvent dicté par le sens du N (avoir du bol, faire la tête, être un bon copain), et c'est plutôt la personne et le temps qui sont informativement pertinents (la tête que tu fais vs la tête qu'il ferait). Il peut cependant arriver que la relative transmette davantage d'information. Dans l'exemple suivant, la charge informative est notamment apportée par le SP complément :

(24) Le divin attachement que je nourris pour Cydalise, maintenant qu'elle échappe aux prises de mon lunaire entendement ! [Laforgue, Imitation Notre-Dame la Lune, 1886, f]

Dans notre structure, le SN défini réfère à un objet-de-discours présenté comme déjà connu, i.e. déjà validé dans la mémoire discursive. Ainsi, la formule semble moins faite pour informer que pour s'exclamer sur une information déjà partagée. Elle attire l'attention sur le caractère exceptionnel, admirable ou simplement remarquable d'une information publiquement valide. L'exemple suivant en montre bien le mécanisme :

(25) Maintenant, parce qu'il a chaud de s'être sottement remué, il s'est découvert jusqu'aux genoux, il montre sans honte son organe, son affreux organe. Oh, cette horreur et cette peur de cet organe qu'il exhibe grossièrement, dont il est fier sans doute, oh que c'est laid et vulgaire, et canin, oui. [Cohen, Belle du Seigneur]

L'exclamative réinvestit des contenus préalablement validés : cet organe qu'il exhibe grossièrement reformule il montre sans honte son organe, et cette horreur fait écho à l'épithète affreux 6 . Elle exprime une réaction affective à un événement préalablement décrit ${ }^{7}$.

Il faut toutefois corriger un peu cette présentation. L'impression selon laquelle le SN ferait l'objet d'un consensus partagé se vérifie pour de nombreux exemples, comme (15) ou (25), mais les cas où l'information véhiculée par le SN n'est pas validée dans le savoir partagé semblent pourtant très fréquents. L'exemple suivant en témoigne; il a été produit par une mère décrivant la mauvaise volonté manifestée par sa fille au moment de se rendre à un cours supplémentaire de violoncelle :

(26) la GUEUle qu'elle tirait c'était imPREssionnant [oral, à la volée, 19.03.2011]

L'allocutaire de l'énoncé n'a pas vu la «gueule » de la fille au moment décrit, et c'est bien l'énoncé qui lui en fait part. Il reste que cette information (elle "tirait la gueule ») est présentée par le SN défini comme si elle était déjà partagée. Seul le caractère impressionnant de la scène, communiqué après la construction nominale, est présenté comme une information nouvelle et rhématique.

De manière comparable, dans l'exemple (10), si la situation d'énonciation est commune aux quatre joueurs de la partie de cartes, le choix du SN le bol témoigne d'une appréciation polémique de cette situation. Ce qui est partagé par tous les joueurs, c'est le résultat de la partie, les péripéties de son déroulement, etc. Le fait d'imputer son issue à la chance des gagnantes n'est certainement pas un jugement aussi consensuel. Il est probable que celles-ci mettraient plus volontiers le résultat au compte de leur habileté. L'emploi du SN défini le bol relève donc d'un «coup de force » présuppositionnel (Ducrot 1972) visant à soustraire cette appréciation au débat.

Lorsque la structure apparaît dans un monologue intérieur, il vaut mieux parler d'un contenu validé que partagé. Dans l'exemple (2), Coindet utilise - mentalement - la construction nominale (le bon copain que c'était) pour se réjouir du fait, déjà valide pour lui, que Truchot est un bon copain. En même temps, la 
structure communique au lecteur du roman, par coup de force, que Coindet considère Truchot comme un bon copain.

Le parti que l'on peut tirer de l'implicite pour transmettre des contenus inédits est bien connu. L'un des rendements principaux des présupposés est de permettre la densification de l'information. En présentant comme présupposées des informations non encore négociées (i.e. en violant leurs conditions d'emploi) on fait appel à la coopérativité de l'interlocuteur, lui demandant d'avaliser a posteriori des présupposés qui sont des transgressions de la mémoire discursive. Le fait qu'une information présupposée est censée présente dans la mémoire discursive est un mécanisme manipulable : il est possible de présenter un contenu comme présupposé sans que cela ait été négocié préalablement.

Dans ces structures, la relative à valeur présupposante n'est pas indispensable : en son absence, par exemple dans (16), (22) et (23), la validité de l'objet-de-discours est assurée uniquement par le présupposé existentiel que véhicule le $\mathrm{SN}$.

Une particularité de nos structures est qu'on ne s'y exclame que sur des contenus déjà validés ou présentés comme tels. Tout en s'exclamant sur un objet, on fait admettre sa validité. La modalité exclamative peut être dans ce cas un moyen argumentatif puissant. Ce n'est cependant pas vrai de toutes les exclamatives: d'autres structures, basées sur un SN indéfini, permettent de s'exclamer sur des contenus qu'elles introduisent, et qui ne sont donc pas préalablement valides : J'ai pris un (de ces) $\operatorname{coup}(s)$ de soleil!

\subsection{Constructions apparentées}

Certaines constructions présentent des traits, notamment formels, proches de notre structure. Elles partagent sans doute certains aspects de son fonctionnement. Par souci de définir un objet d'étude aussi homogène que possible, elles ne seront pas prises en compte dans le cadre de cet article.

- On peut compter parmi elles les exclamatives introduites par un SN indéfini :

(27) Une chouette place de pique-nique que voilà ! Située en bordure de forêt, elle compte plusieurs grandes tables abritées par endroits. [presse, La Liberté, 21.08.2010]

- A mentionner également des variantes de « constructions liées », au sens de Rouquier (2002), mettant en jeu un que qui n'est pas un pronom relatif (vs la fine musique que voilà !) :

(28) La fine musique que celle de l'air filtrant entre les branches, un joli bruit aussi que celui de l'eau roulant sur les graviers ! [Cladel, Ompdrailles, 1879, f]

- Parmi les structures exclamatives construites sur un SN suivi de qui, certaines occurrences sont très proches de nos structures, et pourraient sans doute y être intégrées :

(29) La belle fontaine qui nous coule là ! [Nerval, Faust [trad.], 1928, f]

(30) La belle carrière qui s'ouvre devant lui s'il tape avec application. (...) Cette carrière qui s'ouvre devant lui ! [Boudard, La Cerise, 1963, f]

(31) Ces pensées qui traversaient Anne-Marie ! [Pourrat, Les Vaillances, 1930, f]

Dans ces exemples, comme dans la structure avec que, la relative ne sert qu'à confirmer ou à préciser la référence du SN en le mettant en relation avec la situation de discours ou avec un savoir partagé [qui nous coule là (29), qui s'ouvre devant lui (30), qui traversaient Anne-Marie (31)]. D'autres exemples se distinguent de notre structure par le poids informatif plus important de la relative :

(32) Les beaux yeux qui ne demandent qu'à sourire ! [Colette, Claudine à l'école, 1900, f]

(33) Cette famille qui semblait tellement respecter les morts! [Queffélec, Un Recteur de l'île de Sein, 1944, f] 
(34) Ah! Cette passion qui toujours recommence! Ce ciel que l'ombre ceint d'épines chaque soir ! [Rodenbach, Le Règne du silence, 1891, f]

(35) - Tu n'as qu'une envie, c'est de me voir partir, et pourtant tu sais que j'ai besoin de toi. $\S$ Ces derniers mots la mirent hors d'elle. Cet homme qui avait tout le temps besoin d'elle ! (...) $\S$ Elle regarda avec haine cet homme qui choisissait d'avoir besoin d'elle à minuit. $O h$, cette manie qu'il avait de dépendre d'elle pour tout. [Cohen, Belle du Seigneur]

(36) Oui, un coup de fil à cette chère Pénélope, épouse Kanakis, ça se devait. D'ailleurs, d'après le guide mondain, il fallait remercier le lendemain du dîner. Ainsi fit-il. Le téléphone à la Kanakis terminé, il soupira. Ah là là, cette Ariane qui le forçait à raconter des blagues de migraine parce qu'elle ne gobait pas les Kanakis, des amis charmants pourtant. [Cohen, Belle du Seigneur]

Dans ces exemples, la charge exclamative est placée sur la relative. On s'exclame sur le fait que les yeux ne demandent qu'à sourire (32), que la famille respecte tellement les morts (33), que la passion recommence toujours (34), etc. Du fait du caractère obligatoire de la relative, les constructions (32) à (36) ont un fonctionnement qui les rapproche des « démonstratifs cataphoriques » (Gary-Prieur 1998, Kleiber 2004). On peut penser que ces tours sont composés d'une « exclamative Pellaton» sans relative, suivie d'une relative réalisant une prédication seconde : Cette famille (qu'elle avait) qui semblait tellement respecter les morts ! [exemple modifié à partir de (33)]. Le fonctionnement des exclamatives (1) à (26), avec une relative tout à fait optionnelle, nous semble en revanche différent de celui des démonstratifs cataphoriques.

- Dans les exclamatives introduites par ce que, c'est également le contenu de la relative qui est pertinent informativement et qui motive l'exclamation :

$$
\begin{aligned}
& \text { Ce qu'il jacasse ! [Bonnefoy, La Tempête, 1997, f] } \\
& \text { Ce qu'il a proféré, le con ! [Boudard, La Cerise, 1963, f] }
\end{aligned}
$$

Ces exemples manifestent de la sorte, comme les précédents, un fonctionnement qui les distingue de notre structure.

\section{Le fonctionnement exclamatif}

\subsection{Contextes exclamatifs}

Nous avons déjà évoqué à plusieurs reprises la dimension exclamative de ces constructions. On la sent bien intuitivement, mais il convient de l'appréhender plus précisément. A l'écrit, cette dimension est souvent confirmée par la ponctuation: de nombreux exemples sont accompagnés d'un point d'exclamation ${ }^{8}$. En outre, le contexte environnant est souvent exclamatif. On peut souligner que la construction est fréquemment précédée d'un $o h$ !, d'un $a h$ ! ou d'autres interjections, comme euh mais (11)-(12), putain (13), etc. :

(39) Oh! Les cruelles heures que vous m'avez fait passer! Oh! Les effroyables tortures que vous m'avez fait subir, quand je voyais verser dans votre verre le poison mortel, quand je tremblais que vous n'eussiez le temps de le boire avant que j'eusse celui de le répandre dans la cheminée ! [Dumas père, Le Comte de Monte-Cristo, 1846, f]

Des prédicats verbaux exprimant l'intensité (crier) sont bien représentés dans le cotexte :

(40) Comment ! c'est toi ? cria Mistral en me sautant au cou; la bonne idée que tu as eue de venir ! (...) [Daudet, Lettres de mon moulin, 1879, f]

(41) Ce fut le tour de la mère de se redresser et de crier : - Tu vois bien! Les bêtises que tu fais! En cassant ton carreau, elle s’est coupée ! [Hugo, Les Misérables, 1862, f] 
La présence d'hyperboles n'est pas rare :

(42) Vous me traitez cent fois trop bien, mes braves gens, répondit Marcelle en se hasardant sur la planche qui servait de pont, avec Édouard dans ses bras, pour aller les rejoindre ; jamais je n'ai passé une si bonne nuit, jamais je n'ai vu une aussi belle matinée que chez vous. Les belles truites que vous prenez là, monsieur le meunier! Et vous, la mère, le beau lait blanc et crémeux! Vous me gâtez, et je ne sais comment vous remercier. [Sand, Le Meunier d'Angibault, 1845, f]

(43) Celle-ci (il me montrait une adorable blonde sur Paris-Match), tu ne peux t'imaginer ce qu'elle est vicieuse. Ah! les nuits que nous avons passées! à en mourir ! Je n'en pouvais plus ! [Boudard, La Cerise, 1963, f]

La construction semble donc apparaître volontiers dans des contextes exclamatifs. Il reste à démontrer qu'elle est en elle-même exclamative.

\subsection{La question du haut degré}

Les phénomènes exclamatifs sont traditionnellement décrits en faisant appel au " haut degré » d'une propriété exprimée dans l'énoncé (inter alia Milner 1978, Rivara 1979). Cette description convient-elle à nos exemples? Il paraît nécessaire de distinguer plusieurs cas.

- Le haut degré peut être explicitement exprimé par l'épithète qualifiant le $\mathrm{N}$, comme divin dans (24). Il peut l'être par une expansion du N, comme de merde dans (46), etc. Il peut l'être également par le sens du N lui-même, comme horreur dans (14) et (25), désastre dans (44). On verra infra (§ 4.4.1) que dans certains cas le haut degré peut aussi être exprimé ou suggéré par les prédicats qui apparaissent à la suite de notre construction ; tu verses dans (46), c'était impressionnant dans (47).

- Dans d'autres exemples, le haut degré demeure implicite et doit être reconstruit. Un énoncé comme Le potin que ça faisait... (15) suggère vraisemblablement que le bruit était très gênant, voire insupportable. De même on comprend dans (10) et dans (11) que le bol et le temps dépassent les normes usuelles et admissibles.

- La propriété exprimée par l'énoncé ne se prête pas toujours à la reconstitution d'un « haut degré ». Dans (40) et (44), il n'est pas certain que l'épithète bonne exprime un haut degré, ni que l'énoncé invite à calculer un degré plus haut :

(44) La bonne mine qu'on a avec nos bagages de zonards en exode, nos malles à quatre nœuds de clodos. On attend comme d'habitude trois quatre plombes devant la grille de notre nouvelle division. Le désastre que ça fout dans la routine ces mutations massives! [Boudard, La Cerise, 1963, f]

L'énoncé (40) n'invite probablement pas à reconstruire un degré extrême de la qualité exprimée par bonne (une idée supérieurement bonne ?) et on l'interprète plutôt comme : cette bonne idée me fait un grand plaisir. Dans (1), le beau soleil qu'il faisait dehors est un SN thématique qui laisse au lecteur la liberté de construire une prédication rhématique (c'était magnifique ou cela remplissait Coindet d'aise, etc.). Le processus interprétatif convoque-t-il pour autant un haut degré ? S'il y a un haut degré, ce n'est pas dans la propriété exprimée par l'énoncé lui-même, mais plutôt dans une prédication que l'on reconstruit à partir de l'énoncé. Non seulement le haut degré est à reconstituer, mais également la propriété qui en est affectée.

Les exemples introduits par des SN démonstratifs présentent à cet égard une situation légèrement différente. Dans certains cas, c'est bien une valeur de haut degré que l'énoncé invite à reconstruire. Dans (20), la référence à ce gros dodo évoque un sommeil d'une durée supérieure aux normes ordinaires ou aux habitudes de l'enfant. Avec d'autres exemples, cependant, le contenu implicite à reconstruire n'est pas l'expression d'un degré élevé. Pour les exemples (18) et (19), on ne voit pas bien quel haut degré pourrait s'appliquer à la fadeur ou à la manière évoquées. Il s'agit plutôt d'une fadeur et d'une manière 
singulières, uniques en leur genre. Le fonctionnement particulier de ces structures à SN démonstratif est développé dans le paragraphe suivant.

\subsection{Démonstratifs : l'hypothèse de l'unicité de la qualité exprimée}

Observons ce passage du Planétarium de Sarraute, qui présente de nombreuses occurrences de notre structure $^{9}$ :

(45) Cette illumination qu'elle avait eue... (...) Cette impatience tout à l'heure, cette excitation, quand ils l'ont apportée, quand ils enlevaient avec précaution la bâche qui l'enveloppait... ces gestes délicats, précis et calmes qu'ils ont... (...) cette frayeur quand, assise sous le séchoir, elle avait aperçu juste devant elle une porte arrondie en bois apparent, ça faisait d'un toc... (...) Cette excitation délicieuse, cette confiance, cette allégresse qu'elle sent tandis qu'elle monte l'escalier, sort la clé de son sac (...) cette confiance absurde, ce crédit qu'elle fait aux gens... (...) ils ont cet air imperturbable, ces gestes lents, ce calme professionnel du médecin, tandis que la famille anxieuse attend... « (...) c'est une question d'habitude, vous vous y ferez, vous verrez... c'est très bien, c'est joli... il n'y a qu'à tout laisser comme ça... » Ce ton protecteur qu'ils prennent, ces airs familiers... les voilà déjà qui s'installent en vainqueurs, en prennent à leur aise, la soldatesque avinée, les soudards lui tapotent la joue, lui pincent le menton... (...) Tous ces efforts pour rien... Ces espoirs... cette lutte (...) Cette façon brutale qu'elle a de vous saisir par la peau du cou et de vous jeter là, au milieu de la piste, en spectacle aux gens... Ce manque de délicatesse chez elle, cette insensibilité... Mais c'est sa faute, à lui aussi, il le sait. C'est toujours ce besoin qu'il a de se faire approuver, cajoler... (...) Mais quel air renfrogné tout à coup, quelle moue dégoûtée... Quelle mouche le pique ?... Ce petit ton sec qu'il prend pour refuser, ce regard moqueur... (...) Qu'elle laisse donc ce garçon tranquille. Il a raison, ce petit... C'est inouï, cette insensibilité, cette grossièreté... depuis trente-cinq ans qu'ils sont mariés (...) Elle a raison, il vous empêche de respirer, il vous étouffe - un éteignoir... Ces façons grossières qu'il a de vous rabrouer... rien que de se le rappeler, ça donne chaud... chaque fois qu'on s'excite un peu autour de lui - ce haussement d'épaules impatient... (...) Mais tu ne peux pas t'imaginer ce qu'ils ont fabriqué (...) Viens voir, c'est inouï ce qu'ils ont fabriqué (...) Une idée folle qu'elle avait eue tout à coup... (...) Mais c'était ça, justement, ce petit défaut, ce minuscule accroc, cette petite verrue sur la face de la perfection... [etc.] [Sarraute, Le Planétarium, in Cuvres complètes, 341-355]

Avec les démonstratifs, comme avec le défini, le prédicat implicite peut éventuellement être associé à une idée de haut degré, mais il cible plus fréquemment le caractère particulier, voire unique de la qualité décrite. Au début de (45), cette illumination n'est sans doute pas extrême, mais elle est probablement unique en son genre, reconnaissable entre toutes... Au lieu d'exprimer un « degré ineffable » (Rivara 1979), ces exclamatives soulignent plutôt le caractère singulier, spécifique, inédit, de l'illumination, de l'excitation, de l'allégresse, etc. Cf. <cette illumination bien particulière>, $<c e$ ton protecteur qui n'appartient qu'à eux $>$. Dans nos exemples, on l'a vu, il y a moins d'évaluatifs avec le démonstratif qu'avec l'article défini, donc moins de prédicats gradués, ce qui explique en partie cet état de fait ${ }^{10}$.

Cet effet de sens n'est pas étranger au sémantisme du SN démonstratif, en comparaison avec celui du défini. Nous avons mentionné supra $(\S 3.1)$ que le SN défini présente le référent comme déjà connu. C'est également vrai du SN démonstratif mais, pour celui-ci, le référent est de plus doté d'une saillance cognitive élevée, on peut l'identifier de manière évidente dans la situation partagée ou dans le cotexte proche. Cette différence de fonctionnement entre les SN définis et démonstratifs se manifeste en outre dans le fait qu'avec les premiers, c'est le $\mathrm{N}$ qui permet l'identification du référent (regarde la vache), tandis qu'avec les seconds, c'est le déterminant démonstratif lui-même qui donne l'accès au référent visé (regarde cette vache). A côté de ses emplois habituels, il existe des cas dans lesquels un SN démonstratif ne peut être identifié ni dans la situation partagée ni dans le cotexte environnant: 
Un syntagme nominal démonstratif peut référer in absentia, c'est-à-dire en l'absence de toute désignation antécédente de son référent et sans que celui-ci soit présent dans la situation d'énonciation. [Apothéloz 1995 : 35]

Il s'agit alors d'un fonctionnement du démonstratif connu notamment sous le nom de «déixis mémorielle », dont Apothéloz (1995) donne ici une description :

\begin{abstract}
On s'accorde à décrire ce type de $\mathrm{SN}$ démonstratif en disant qu'il consiste à évoquer un référent dont l'évidence est telle, pour le locuteur, qu'il équivaut à un référent qui viendrait juste d'être évoqué dans le même texte. [...] cet emploi de la référence démonstrative s'accompagne parfois d'un effet de sens particulier: il donne au destinataire l'impression d'avoir un accès immédiat à l'état cognitif dans lequel se trouve un tiers, que ce dernier soit l'énonciateur ou un personnage explicitement évoqué. [Apothéloz 1995 : 35-36]
\end{abstract}

C'est un fonctionnement de ce genre qu'exploitent nos exemples, et qui permet de rendre compte de l'effet de sens mentionné ci-dessus. Dans une exclamative comme ce ton protecteur qu'ils prennent (45), le fait que le démonstratif évoque un référent immédiatement accessible et évident pour l'allocutaire va de pair avec le fait que ce référent soit envisagé dans sa particularité et son unicité. Autrement dit, le démonstratif met en évidence l'unicité d'un objet montré, alors que le défini présuppose l'unicité d'un objet (et rien de plus). On interprète ce ton protecteur comme <le ton protecteur qui leur est propre, entre tous les tons protecteurs qu'il est possible d'adopter>. C'est bien la situation de connivence installée par le démonstratif entre le locuteur et l'allocutaire, ou, pour le dire dans les termes d'Apothéloz (1995), l'accès immédiat de l'allocutaire à l'état cognitif du locuteur, qui est à l'origine de cet effet de sens ${ }^{11}$.

Ce fonctionnement peut sans doute expliquer également le fait que, comme nous l'avons dit au $\S 2.2 .2$, les SN démonstratifs puissent s'accommoder plus facilement de l'absence de la relative que les définis. Les éléments signalés en italiques dans (45) illustrent ce cas de figure. La valeur «mémorielle»du démonstratif rend la présupposition véhiculée par la relative encore plus redondante, et par conséquent plus facultative, le référent étant présenté comme une donnée d'évidence pour l'allocutaire. Au contraire, dans les constructions à SN défini, le référent, s'il est présent dans le savoir partagé, ne l'est pas de manière aussi saillante, aussi évidente, ce qui rend la présence de la relative plus naturelle. On voit que le choix du défini ou du démonstratif permet de moduler le point de vue sur le référent.

Ces observations permettent de confirmer que nos constructions n'évoquent pas toutes l'idée d'un haut degré. Certains des énoncés construits autour d'un SN démonstratif suggèrent plutôt le caractère particulier, " unique en son genre » d'un fait ou d'une propriété. Ces effets de sens peuvent être regroupés sous la notion d'écart vis-à-vis d'une norme ordinaire, autrement dit sous la notion d' « extra-ordinaire » (Marandin à par., § 7, Remarque). Ce que nos structures exclamatives expriment ou impliquent, c'est le caractère hors norme d'un fait.

\title{
4.4 L'attente d'une prédication
}

\subsubsection{Prédication exprimée et prédication implicite}

Les structures étudiées déclenchent une attente. Les énoncés se présentent sous la forme averbale d'un SN défini, correspondant à un objet-de-discours dont l'existence est présentée comme présupposée, avec par conséquent une valeur thématique (les présupposés n'ont pas contexte commun avec les rhèmes). Ainsi l'énoncé nominal ouvre l'attente d'une prédication rhématique.

Dans certains cas, comme dans les exemples (46) à (48), cette prédication est explicitement formulée juste à la suite de la construction (ci-dessous, elle est signalée en italiques); voir également (43) ( $\grave{A}$ en mourir! Je n'en pouvais plus!) :

(46) non mais les passes de MERde qu'ils font $+t u$ verses [oral, à la volée, énoncé par un spectateur d'un match de football] $]^{12}$ 
(47) la GUEUle qu'elle tirait c'était imPREssionnant [oral, à la volée, 19.03.2011, une mère à propos de sa fille fâchée de devoir se rendre à un cours supplémentaire de violoncelle]

(48) Toutes les histoires qu'on a avec les créanciers! C'est à se tordre, si vous saviez! [Claudel, Partage de midi [version pour la scène], 1949, f]

Dans ces exemples, l'élément thématique ' $\mathrm{SN}+$ relative' est suivi d'une prédication hyperbolique, qui laisse entendre comme étaient renversante la médiocrité des passes (46), impressionnante la bouderie de la petite fille (47), risibles ou dérisoires les histoires de créanciers (48), et épuisantes les nuits passées avec une adorable blonde (43).

Cependant, dans la majorité des exemples, le cotexte ne permet pas d'assouvir l'attente. En l'absence d'une prédication explicite telle que dans (46)-(48), la structure invite à reconstruire une composante prédicative implicite visant à combler cette attente.

\subsubsection{Rapprochement avec des énoncés « incomplets »}

La comparaison avec des constructions ressemblantes peut confirmer l'idée que notre structure ouvre l'attente d'une prédication. Il s'agit de structures dans lesquelles un SN défini suivi d'une relative ne fait pas l'objet d'un énoncé nominal, mais est intégré dans une construction verbale. En voici un sous la forme d'une protase hypothétique :

(49) Si tu savais le peu que c'était dans ma vie ! [France, Le Lys rouge, 1894, f]

Cet énoncé en si provoque l'attente d'une apodose qui reste implicite et que le lecteur peut reconstruire en contexte. Une proposition au conditionnel à valeur hypothétique peut fonctionner de la même manière qu'une hypothétique en si :

(50) putain tu aurais vu le cul qu'elle a !!!!! [www]

Les cas où le SN est introduit par avec, là aussi sans élément recteur, relèvent d'un fonctionnement comparable :

(51) - Alors draguons! il faisait. On peut bien se taper une bonniche à l'œil, non? Merde avec la classe qu'on a ! [Bertrand Blier, Les Valseuses, 1972, f]

Le segment $\{$ avec $+\mathrm{SN}\}$ est manifestement un élément régi. En l'absence d'un bon candidat pour le recteur, on est conduit à penser que celui-ci est ellipsé, et doit être reconstruit par inférence (par exemple : on aura forcément un grand succès).

\subsubsection{Rapprochement avec des énoncés interrogatifs ou jussifs}

Nos SN suivis de relatives se rencontrent également intégrés dans des énoncés «exclamatifs » en tu te rends compte. Comme d'autres exclamatives, ceux-ci sont probablement des interrogatives déguisées :

(52) Tu te rends compte de la chance qu'on a ! [Beauvoir, Les Mandarins, 1954, f]

Ces interrogatives particulières mettent en doute que le destinataire puisse se rendre compte d'un fait excédant les normes coutumières, et suscitent de sa part une réaction d'approbation de ce caractère extraordinaire. L'exemple suivant montre un cas où cette approbation (en italiques) est exprimée par l'énonciateur même de la question :

(53) Tu te rends compte les larmes qu'il faudrait pour pleurer équitablement suivant l'ampleur du désastre ? C'est pas humain. [Thérame, Bastienne, 1985, f]

Il en va de même avec des énoncés interro-exclamatifs en t'as vu, tu vois, etc.

(54) T'as vu le mal qu'on a avec l'excision ! [Thérame, Bastienne, 1985, f] 
Avec des énoncés n'exigeant pas à strictement parler une complétion, on peut penser à un fonctionnement du même genre ; ils peuvent également inviter à reconstruire un implicite :

(55) Il faut voir le monde qu'il y a ! [Vallès, L'Enfant, 1879, f]

L'exclamative de (55) suscite l'élaboration d'une forme de prédication hyperbolique justifiant l'injonction de voir le monde qu'il y a (par ex. ça dépasse l'imagination).

On peut noter finalement qu'en ne gardant que le SN des exemples cités sous $\S \S 4.4 .2$ et 4.4.3, on obtient des exclamatives crédibles, tout à fait conformes à notre structure : le peu que c'était dans ma vie!; le cul qu'elle a!!!!!; tous les Allemands qu'il y a dans la ville!; la chance qu'on a!; les larmes qu'il faudrait; le mal qu'on a avec l'excision! ; le monde qu'il y a ! La parenté de fonctionnement entre les structures verbales et nominales apparaît ainsi clairement.

\section{Hypothèses}

\subsection{Structures informationnelle et prosodique}

Sur la base des exemples présentés supra, nous formulons l'hypothèse suivante : le fonctionnement exclamatif des constructions étudiées s'explique par l'ouverture d'une attente de prédication rhématique à caractère souvent hyperbolique. Cette prédication est parfois exprimée dans le cotexte droit, mais elle est le plus souvent à reconstruire. De manière plus générale, il paraît légitime de penser que l'un des procédés exclamatifs consiste à n'exprimer que la partie thématique d'un énoncé ${ }^{13}$. Le locuteur renonce à une formulation qui dépasse peut-être ses moyens descriptifs ou expressifs, ou du moins il juge argumentativement plus efficace de laisser le contenu hyperbolique à l'imagination de son destinataire.

Ainsi s'explique pourquoi un énoncé nominal comme le bol qu'elles avaient! (10) est exclamatif, alors qu'un énoncé verbal comme elles avaient $d u$ bol ne l'est pas. Ce dernier décrit un état de fait, au moyen d'un énoncé complet. Au contraire, la construction nominale (10), par une certaine incomplétude, invite à reconstruire une prédication rhématique. La construction verbale pourrait devenir exclamative, par exemple, avec le recours à un SN indéfini : elles ont eu un (de ces) bol(s) ! Cette formulation engendrerait alors le besoin de calculer un contenu implicite destiné à lever l'indétermination de l'objet désigné par le SN (un bol inoü / qu'on ne voit que rarement / comme on n'en a jamais vu). On le voit, diverses structures exclamatives fonctionnent en suscitant une attente, par différents moyens, au nombre desquels on peut mentionner l'incomplétude syntaxique (si tu savais le bol qu'elles avaient!), la sousdétermination d'un objet-de-discours (elles avaient un de ces bols!), ou encore, comme dans notre structure, le simple signalement d'un référent dépourvu de prédication à son sujet (le bol qu'elles avaient!).

La prosodie fait également partie des moyens mis en œuvre. Il faut signaler que le SN de nos structures bénéficie généralement d'un fort accent expressif (intensité et allongement, notamment). Nous l'avons constaté dans les exemples oraux, et cela correspond à la réalisation la plus plausible des exemples écrits ${ }^{14}$. Cette accentuation d'un contenu thématique est étonnante, voire paradoxale. Elle joue sans doute un rôle dans le fonctionnement expressif de la structure, en contribuant à provoquer l'attente d'un rhème hyperbolique. Un $\mathrm{SN}$ isolé qui ne serait pas affecté de cette accentuation serait également compris comme thématique, mais sans dimension exclamative ; il serait probablement interprété comme une sorte de titre pour la séquence qui suit (comparer La VACHE! et La vache.)

\subsection{Extension à d'autres structures exclamatives}

Notre hypothèse semble pouvoir s'appliquer à d'autres exclamatives :

(56) Comme elle a grandi !

((Qu'est-)ce) qu'il est intelligent !

Quelle chaleur ! [< Riegel \& al. 2009] 
Il paraît légitime de considérer que ces énoncés n'expriment pas explicitement une hyperbole, mais qu'ils suscitent l'attente d'une prédication hyperbolique. Cette dernière peut d'ailleurs être verbalisée :

(57) Comme elle a grandi ! C'est incroyable !

((Qu'est-)ce) qu'il est intelligent ! C'est fou !

Quelle chaleur! Je n'en reviens pas!

A l'appui de cette idée, on peut observer que, tout comme nos structures à SN défini, ces formulations exclamatives peuvent également se trouver intégrées dans des constructions verbales suscitant l'attente d'une complétion (v. supra § 4.4.2). En témoignent les exemples suivants :

(58) Si vous saviez comme c'est difficile ! [Winckler, La maladie de Sachs, 1998, f]

(59) - Si tu savais ce que mon paternel me fait chier! [Schreiber, Un silence d'environ une demi-heure, 1996, f]

(60) «Oh! Papa, si tu savais quel travail nous avons eu ces derniers jours!» [Camus $<$ Marandin, à par.]

(61) Tu te rends compte comme c'est humiliant ce que tu fais là ? [Pagnol, Marius, 1931, f]

(62) Tu ne t'imagines pas quel poète c'est que Ronsard. Quel poète ! Quel poète ! Quelles ailes ! C'est plus grand que Virgile et ça vaut du Goethe (...) [Flaubert, Correspondance, 1852, f]

L'exemple de Flaubert est intéressant à cet égard. Il commence par une structure verbale enchâssant une proposition en quel, sous la forme d'une question indirecte. Cette structure ouvre l'attente d'une prédication (puisque le destinataire n'imagine pas quel poète est Ronsard, il peut légitimement attendre que le locuteur le lui dise). Ensuite, trois exclamatives en quel font monter encore cette attente, avant que la prédication hyperbolique, qui aurait pu rester implicite, vienne la résoudre.

Depuis longtemps, les exclamatives en tellement, en tant et en si ont également été traitées comme des structures incomplètes (Milner 1978, Rivara 1979, Kerfelec 2009, etc. ; v. aussi la note 13) :

(63) Il fait si beau!

Il fait tellement beau!

Il y a tant de soleil !

Ce type d'énoncé provoque l'attente d'une proposition consécutive, pour laquelle on pourrait proposer cette paraphrase générique approximative : que c'en est extraordinaire. Parfois, cette attente est comblée par une prédication exprimée :

(64) Comment, Monsieur ! s'écria Bazin, un poème se vend si cher ! C'est incroyable ! [Dumas, Les Trois mousquetaires, 1844, f]

Un certain nombre de formules exclamatives paraissent donc fonctionner en ouvrant l'attente d'un prédicat.

\subsection{Un fonctionnement exclamatif différent}

Il reste à mentionner une structure apparentée aux "exclamatives Pellaton » mais se comportant de manière différente, et même radicalement opposée. Dans cette structure, la prédication est exprimée d'emblée - contrairement au fonctionnement étudié au $\S 4.4$-, et notre énoncé nominal ne sert qu'à indiquer ou à confirmer a posteriori à quel thème la prédication en question se rapporte :

(65) Entré dans l'ascenseur, il se contempla dans la glace. Adrien Deume, fonctionnaire international, confia-t-il à son image, et il sourit. Oui, géniale, cette idée qui lui était venue hier de fonder une société de conférences littéraires. Ce serait le bon truc pour augmenter son capital de relations. [Cohen, Belle du Seigneur] 
(66) (...) oh tellement comique le genre taureau pressé soucieux qu'il prend à ces momentslà $(. .$.$) [Cohen, Belle du Seigneur]$

(67) (...) affreux, les seins qu'elle doit avoir (...) [Cohen, Belle du Seigneur]

(68) Insensé, les paroles que j'arrive à prononcer, tout en pensant à autre chose. [Triolet, Le Premier accroc coûte deux cents francs, 1945, f]

(69) C'est fou le nombre de personnes que j'aurai été obligé de consoler de ce qui nous arrivait ! [Forest, L'enfant éternel, 1997, f]

A la différence de nos structures, la séquence ' $\mathrm{SN}+$ relative' ne provoque pas d'attente, puisque la prédication est exprimée au préalable. Au point de vue prosodique, ces exemples se distinguent des autres par le fait que c'est la prédication initiale qui est mise en relief prosodiquement, alors que le SN reçoit une prosodie d'élément postfocal confirmatif (Berrendonner 2008).

Cette structure témoigne d'un fonctionnement exclamatif diamétralement opposé à celui que nous avons observé jusqu'ici. Un adjectif ou une courte construction verbale exprime avec emphase une prédication :

(70) - Mirobolant, dit la Squaw.

- Fascinant, tout à fait fascinant, confirma Savorgnan.

- Par surcroît, ajouta Aloysius, il n’y a qu'un «y » : dans « Whisky »!

- Confondant! Saisissant ! Inouï ! [Perec, La Disparition, 1969, f]

Celle-ci peut éventuellement être suivie de la confirmation du fait auquel elle s'applique :

(71) Formidable, en somme, ce qu'il gagnait! [Cohen, Belle du Seigneur]

(72) Ouais génial pour les textes! [courriel, 29.09.11, réponse d'un auteur recevant la nouvelle que ses textes ont été relus, et appréciés par le relecteur]

\section{Conclusion}

Nous avons épuisé tous les "C'est merveilleux!», "C'est incroyable!», «C'est insensé !». Les «quand je pense...», les «si on m'avait dit...» [Dorin, Les Vendanges tardives, 1997, f]

L'étude des structures originales que nous avons appelées « exclamatives de Mlle Pellaton » nous conduit en fin de compte à proposer une hypothèse plus générale concernant le fonctionnement des énoncés exclamatifs. Au sein du domaine - vaste, hétérogène et donc difficile à circonscrire - de l'exclamation semble se détacher une sous-classe d'énoncés particuliers. Ces exclamatives peuvent être décrites comme la réaction expressive à un fait qui s'écarte d'une norme ordinaire selon le jugement subjectif du locuteur $^{15}$. Face à un tel fait, deux procédés exclamatifs sont en concurrence. Le premier, qu'on peut qualifier de «direct», consiste à exprimer un prédicat hyperbolique concernant ce fait (Génial! Super! C'est extraordinaire! C'est fou!), éventuellement suivi de la confirmation du thème auquel il se rapporte (C'est incroyable, ce que tu me dis là !). Le second est un procédé "indirect " par lequel le locuteur énonce le fait hors norme (Ce match!), un trait associé à celui-ci (Le bol qu'elles avaient!) ou toute autre formule destinée à l'évoquer ou le suggérer ( $\mathrm{Si}$ tu savais...), dans une forme suscitant l'attente d'un prédicat hyperbolique, celui-ci pouvant être ensuite explicitement verbalisé, ou rester implicite.

Notre hypothèse, qu'il faudra encore examiner en détail pour les différentes structures concernées, exploite et rejoint plusieurs aspects des descriptions antérieures. Cependant, elle s'en écarte par certains points non négligeables. Nous avons déjà exprimé aux paragraphes 4.2 et 4.3 quelques réticences à considérer que les exclamatives feraient toujours appel à une notion de haut degré ${ }^{16}$. Nous ajoutons encore une réserve à l'égard de la notion d'indicible, souvent invoquée pour justifier l'incomplétude manifestée par les structures exclamatives (v. notamment Rivara 1979 et 1990). La distinction que nous proposons entre deux procédés exclamatifs va à l'encontre de cette notion. En effet, en recourant au 
procédé que nous appelons direct, le locuteur n'hésite pas à exprimer un contenu à caractère hyperbolique (ce qu'il ne pourrait pas faire s'il était indicible); en outre, même dans le cas du procédé indirect, les formulations instaurant l'attente d'une prédication hyperbolique sont parfois suivies de son expression explicite. La notion d'indicible ne nous paraît donc pas pouvoir être généralisée à tous les cas d'exclamation. Dans ce domaine, le non-dit ne relève peut-être pas tant d'une " impossibilité de dire » (Kerfelec 2009) que d'un choix argumentatif entre dire ou ne pas dire.

\section{Bibliographie}

Apothéloz, D. (1995). Rôle et fonctionnement de l'anaphore dans la dynamique textuelle. Genève : Droz.

Berrendonner, A. (2008). Il est beau, le lavabo : il fait problème, cet intonème. In Birkelund, M., Mosegaard Hansen, M. \& Norén, C. (éds), L'énonciation dans tous ses états. Mélanges offerts à Henning Nølke à l'occasion de ses soixante ans, Bern : Peter Lang, 669-687.

Ducrot, O. (1972). Dire et ne pas dire. Paris : Hermann.

Gary-Prieur, M.-N. (1998). La dimension cataphorique du démonstratif. Etude de constructions à relative. Langue française, 120, 44-50.

Groussier, M.-L. (1995). L'exclamatif, l'intensif et le focalisé. Faits de langues, 6, 217-229.

Kerfelec, V. (2009). L'exclamation en français et en anglais ; Formes, sens, effets. Aix-en-Provence : Publications de l'Université de Provence.

Kleiber, G. (2004). Sémantique, référence et discours : le cas des démonstratifs cataphoriques spécifiques. In Auchlin, A. et alii (éds), Structures et discours. Mélanges offerts à Eddy Roulet, Québec : Editions Nota bene, 231-245.

Marandin, J.-M. (à par.). Le type exclamatif. In Godard D., Abeillé A. \& Delaveau A., (éds), Grande Grammaire du Français.

Milner, J.-C. (1978). De la syntaxe à l'interprétation : quantités, insultes, exclamations. Paris : Seuil.

Riegel, M., Pellat, J.-C. \& Rioul, R. (2009). Grammaire méthodique du français. Paris : PUF.

Rivara, R. (1979). La quantification corrélative. Sigma, 4, 79-107.

Rivara, R. (1990). Le système de la comparaison. Paris : Éditions de Minuit.

Rouquier, M. (2002). Les constructions liées : "c'est une saine occupation que l'horticulture". Bulletin de la Société de Linguistique de Paris, XCVII-1, 153-186.

Vanderbauwhede, G. (2011). Le déterminant démonstratif en français et en néerlandais à travers les corpus : théorie, description, acquisition. Thèse de doctorat de la Katholieke Universiteit Leuven et de l'Université de Paris Ouest Nanterre La Défense.

\footnotetext{
${ }^{1}$ Cette étude a été élaborée dans le cadre des projets FNS suivants : Modélisation des dépendances syntaxiques en français (subside $\mathrm{n}^{\mathrm{O}} 139584$ ) et L'inversion du sujet clitique en français moderne : variations et fonctions (subside $\mathrm{n}^{\circ}$ 132019).

${ }^{2}$ Dans Frantext, on peut rechercher les cooccurrences d'un SN suivi de que et d'un point d'exclamation : cela n'est guère possible pour les corpus d'oral.

${ }^{3}$ Sans tenir compte d'une éventuelle antiphrase.

${ }^{4}$ Les exemples marqués d'un ' $\mathrm{f}$ ' sont tirés de la base Frantext.

${ }^{5}$ Les petites capitales marquent un accent expressif.

${ }^{6}$ Dans (25), on notera que notre structure précède, avec un parallélisme évident, une autre construction exclamative : oh que c'est laid et vulgaire... (cf. infra, § 5.2)

${ }^{7}$ Pour des exemples apparentés à (25), Vanderbauwhede (2011) parle de « reformulation mémorielle ».
} 


\begin{abstract}
${ }^{8}$ Le fait qu'on ait beaucoup d'exemples ponctués par un point d'exclamation est peut-être un biais de la méthode de recherche, mais le fait que l'on tombe sur notre structure en appelant le point d'exclamation confirme l'intuition qu'elle est ressentie comme exclamative par ses utilisateurs.

Les constructions (1) et (2) sont assez peu prototypiques des faits que nous avons recueillis dans notre corpus : il s'agit de monologues intérieurs au discours indirect libre, ce qui explique peut-être qu'ils soient ponctués d'un simple point. Les exemples sans point d'exclamation observés dans le Planétarium sont également des pensées rapportées. Nous verrons infra que la structure, quelle que soit la ponctuation, est néanmoins fondamentalement exclamative.
\end{abstract}

${ }^{9}$ Dans (45), en gras, notre structure. En italiques, la structure sans la relative ; cf. exemples (22) et (23). Soulignées, les relatives de constructions apparentées, avec un prédicat verbal ou un indéfini.

${ }^{10}$ A cet égard, l'exemple de Sarraute est un peu atypique, et témoigne d'une écriture très personnelle.

${ }^{11}$ Autre fait intéressant, à mettre peut-être en rapport avec l'idée de déixis mémorielle : on observe un pointage déictique sur des éléments situationnels, que ce soit au plan mimo-gestuel (gestes, regard, façon(s), airs dans 45, manière dans 19, sourire dans 21, soupir dans 17, etc.), ou intonatif (ton, à deux reprises dans 45). Voir aussi les nombreux exemples avec les déictiques là (5), (7), (9), (29) et voilà (Les jolis enfants que voilà ! Vigny; Le triste amour que voilà ! Apollinaire).

${ }^{12}$ Prosodié en deux morceaux successifs, comme deux exclamatives placées l'une à la suite de l'autre, avec un accent d'intensité et un allongement sur la voyelle $[\varepsilon]$ de merde. A noter que tu verses est équivalent à tu tombes à la renverse.

${ }^{13}$ Cette hypothèse peut être mise en rapport avec l'idée qui voit l'exclamation comme une corrélation « tronquée » ou « non corrélée » (v. Milner 1978, Rivara 1979, Kerfelec 2009), mais elle permet probablement de décrire davantage de structures, et avec davantage de généralité.

${ }^{14}$ Nos énoncés oraux ayant tous été pris à la volée, nous n'en avons pas d'enregistrement, et ne pouvons donc pas en faire des analyses acoustique instrumentalisées. Nos observations, sommaires, sur la prosodie de ces structures sont donc uniquement perceptives.

${ }^{15}$ D'autres exclamations ne sont pas du tout associées à un fait exceptionnel ou hors norme. Par exemple, certaines semblent motivées par le besoin que le locuteur ressent de défendre ce qui est pour lui une évidence (Bien sûr! Tu parles!)

${ }^{16}$ On peut s'étonner que nous nous opposions à l'idée de haut degré, alors que nous faisons appel à la notion d'hyperbole. Les deux ne se confondent pas, à notre avis. Des qualificatifs comme exceptionnel, incroyable, étonnant peuvent créer un effet d'hyperbole en pointant sur la singularité d'un fait, sans pour autant exprimer le haut degré d'une qualité. 